\title{
EVOLUCIÓN SEDIMENTARIA DEL LAGO KÁRSTICO DE LA PARRA (CUENCA) DURANTE LOS ÚLTIMOS 1600 AÑOS: PALEOHIDROLOGÍA, CLIMA E IMPACTO HUMANO
}

\author{
F. BARREIRO-LOSTRES ${ }^{*}$, A. MORENO', \\ S. GIRALT ${ }^{2}$, B.L. VALERO-GARCÉS ${ }^{1}$ \\ ${ }^{1}$ Dpto. de Procesos Geoambientales y Cambio Global, Instituto Pirenaico de Ecología, CSIC, \\ Campus Aula Dei, Av. Montañana 1005, 50059 Zaragoza. \\ ${ }^{2}$ Instituto de Ciencias de la Tierra Jaume Almera, ICTJA-CSIC, C/ Lluís Solé i Sabarís s/n, \\ 08028 Barcelona.
}

\begin{abstract}
RESUMEN. La laguna de La Parra (39 50', $1^{\circ}$ 52', 1014 m) es un lago pequeño (1 ha), relativamente profundo $(Z \max =17.5 \mathrm{~m})$ de agua dulce $(T D S=356 \mathrm{mg} / \mathrm{L})$, monomíctico y con morfología circular (116 m diámetro), formado en una dolina que interceptó el acuífero regional del Jurásico y Cretácico Medio. Forma parte del complejo kárstico de las Torcas de Cañada del Hoyo constituido por siete lagos y situado en la Cordillera Ibérica (Cuenca, Este de España). La secuencia sedimentaria del lago se ha estudiado mediante técnicas sedimentológicas, microscópicas, geoquímicas y físicas aplicadas a sondeos de gravedad cortos y a un sondeo largo $(6.93 \mathrm{~m})$ que registra la evolución del lago desde su inicio, La secuencia está caracterizada por una gran variabilidad de facies clásticas carbonatadas, controladas principalmente por fluctuaciones en la entrada de aportes detríticos al lago y variaciones en el balance hídrico. La cronología está basada en 7 dataciones de ${ }^{14} \mathrm{C}$ AMS. Los cambios en los ambientes de depósito de La Parra reflejan una amplia variabilidad hidrológica durante los últimos 1600 años. Con anterioridad a la época romana, la dolina de La Parra era una torca seca, y la laguna sólo se desarrolló a partir del siglo IV de nuestra era. La reconstrucción paleohidrológica y paleoambiental muestra una fase árida durante la Anomalía Climática Medieval (ACM) y otra más húmeda durante la Pequeña Edad del Hielo (PEH). La principal afección antrópica en el lago y su entorno se produjo durante la Edad Media (500-1400 DC) y los siglos XVIII- mitad del siglo XX relacionada con cambios en el uso del suelo (trashumancia, deforestación, agricultura) y se refleja en cambios en la tasa de sedimentación y la composición de los sedimentos.
\end{abstract}

Sedimentary evolution of La Parra karstic lake (Cuenca) over the last 1600 years: paleohydrology, climate, and human impact

ABSTRACT. Lake La Parra (39 50', 1 52', $1014 \mathrm{~m})$ is a small (1 ha), relatively deep $(Z \max =17.5 \mathrm{~m})$, freshwater $(T D S=356 \mathrm{mg} / \mathrm{L})$ monomictic lake with a 
circular morphology (116 m diameter), developed in a doline that intercepted the local Jurassic-Cretaceous aquifer. It is part of a karstic lake complex of seven lakes located in the Iberian Range (Cuenca, East of Spain). Sediment cores obtained in the central part of the lake were analysed using sedimentological, microscopic, geochemical and physical techniques. The ca. $7 \mathrm{~m}$ long sequence is mainly composed by clastic carbonated sediments, characterized by a high variability of sedimentary facies, indicative of large fluctuations in the sediment delivery to the lake. Chronology is based on 7 AMS radiocarbon dates. The depositional evolution of La Parra sequence reflects a high hydrological variability during the last 1600 years. Prior to the Roman Epoch, La Parra doline was dry and only became a lake after $4^{\text {th }}$ century $A D$. Shallower conditions and higher sediment input occurred during the Medieval Climate Anomaly (MCA) and more humid phases during the Little Ice Age (LIA). The main human impact in the lake and the watershed environment is related to changes in the land uses (transhumance, deforestation, crops) during the Middle Ages (500-1400 DC) and during $18^{\text {th }}$ - mid $20^{\text {th }}$ centuries.

Palabras clave: Holoceno tardío, facies sedimentarias, geoquímica, lago kárstico, ambientes de sedimentación lacustre, paleohidrología, Península Ibérica.

Key words: Late Holocene, sedimentary facies, geochemistry, karstic lake, lacustrine depositional environments, palaeohydrology, Iberian Peninsula.

Enviado el 5 de noviembre de 2012

Aceptado el 7 de diciembre de 2012

* Correspondencia: Dpto. de Procesos Geoambientales y Cambio Global, Instituto Pirenaico de Ecología, CSIC, Campus de Aula Dei, Av. Montañana 1005, 50059 Zaragoza. E-mail: ferbalos@ipe.csic.es

\section{Introducción}

Recientemente se han llevado a cabo numerosas reconstrucciones climáticas en el área mediterránea basadas en estudios multidisciplinares (isótopos, polen, geoquímica, sedimentología) de sistemas lacustres, que han demostrado ser archivos excepcionales de la evolución ambiental y climática a escala regional (ver referencias en Valero-Garcés y Moreno, 2011; Moreno et al., 2012; Roberts et al., 2012). En la Península Ibérica, los lagos están localizados en una gran variedad de condiciones geográficas, climáticas y ecológicas. Existen lagos de montaña formados por la actividad glaciar (Enol, Sanabria) (Moreno et al., 2009; López-Merino et al., 2011); lagos kársticos cuyo origen es debido a la acción exokárstica (Taravilla, Somolinos) (Moreno et al., 2008; Currás et al., 2012) o a la disolución de evaporitas o carbonatos (Banyoles, Moncortés, Zoñar, Estanya) (Höbig et al., 2012; Corella et al., 2012; Martín-Puertas et al., 2009; Morellón et al., 2011); también se encuentran lagos salinos efímeros en las cuencas de los ríos Ebro, Duero y Tajo, originados por la combinación de procesos erosivos y kársticos (por ejemplo, salinas de Bujaraloz) (González-Sampériz et al., 2008). En todas las secuencias 
lacustres se han descrito cambios climáticos significativos en el Holoceno tardío, particularmente durante el Periodo Húmedo Ibero-Romano, la Anomalía Climática Medieval y la Pequeña Edad de Hielo (Valero-Garcés y Moreno, 2011; Moreno et al., 2012).

En este trabajo se ha llevado a cabo un estudio sedimentológico y geoquímico de alta resolución en sondeos obtenidos en la laguna kárstica de La Parra, situada en la Cordillera Ibérica (NE de España, provincia de Cuenca). Facies y composición química han estado influenciadas tanto por el clima como por el impacto humano en el área (ganadería, agricultura, fuegos), muy intenso al menos desde la época medieval. El robusto modelo cronológico proporcionado por técnicas de datación de radiocarbono AMS, ha permitido describir los cambios en las condiciones del lago, su cuenca de recepción e investigar la compleja interrelación entre el clima y las actividades humanas desde el final del Periodo Romano.

\section{Zona de estudio}

La laguna de La Parra ( $39^{\circ} 50^{\prime} \mathrm{N}, 1^{\circ} 52^{\prime}$ E, 1014 m.s.n.m.) es una dolina que pertenece al sistema de lagos kársticos de Cañada del Hoyo (Fig. 1), formada por procesos de disolución y diaclasado (Alonso, 1986) en calizas y dolomías cenomanienses-turonienses, afectando también a materiales jurásicos. La falla de Valdemoro y un anticlinal regional de eje principal NW-SE han sido claves en el desarrollo de este sistema kárstico (Eraso et al., 1979; Carmona y Bitzer, 2001).

Climáticamente, se encuentra en un contexto Mediterráneo, modulado por influencias continentales con un elevado contraste diario y estacional de las temperaturas (temperatura media desde $4^{\circ} \mathrm{C}$ en enero hasta $23^{\circ} \mathrm{C}$ en julio). La precipitación media anual se sitúa en 542 mm, siendo julio el mes más seco y octubre el más húmedo.

La vegetación local está dominada por Pinus nigra, Quercus faginea, Quercus ilex rotundifolia, Juniperus thurifera, Buxus sempervirens y Quercus coccifera. Las depresiones y zonas bajas están destinadas al cultivo de cereal.

La laguna de La Parra tiene morfología circular (113 m de diámetro, 1 ha de superficie), y una profundidad máxima de $17.5 \mathrm{~m}$. No tiene entradas o salidas de agua superficiales permanentes, pero está conectada hidráulicamente (Carmona y Bitzer, 2001) con las aguas subterráneas del acuífero local carbonatado de la Formación de Dolomías de Cortes de Pallás (Gil et al., 2004). Un escarpe en dolomías del Cretácico Superior (Fm Dolomías de la Ciudad Encantada) rodea la laguna desde su parte oeste hasta el sureste.

El comportamiento hidrológico de la laguna está controlado por: i) una cuenca de recepción pequeña ( 10 ha); ii) la relación entre el acuífero local y el regional, iii) un régimen de precipitaciones fuertemente estacional; iv) una alta evaporación y v) fluctuaciones del nivel freático del acuífero regional por extracciones agrícolas (pozos). Análisis de las aguas llevados a cabo en 2010 indican una composición dominada por bicarbonatos $(4.78 \mathrm{meq} / \mathrm{L})$ y magnesio $(3.96 \mathrm{meq} / \mathrm{L})$, coherente con la litología circundante. La conductividad eléctrica oscila desde los $305 \mu \mathrm{S} / \mathrm{cm}$ en superficie hasta los 356 $\mu \mathrm{S} / \mathrm{cm}$ a $16 \mathrm{~m}$ de profundidad. La alcalinidad se sitúa en torno a los $6.0 \mathrm{meq} / \mathrm{L}$, siendo el 


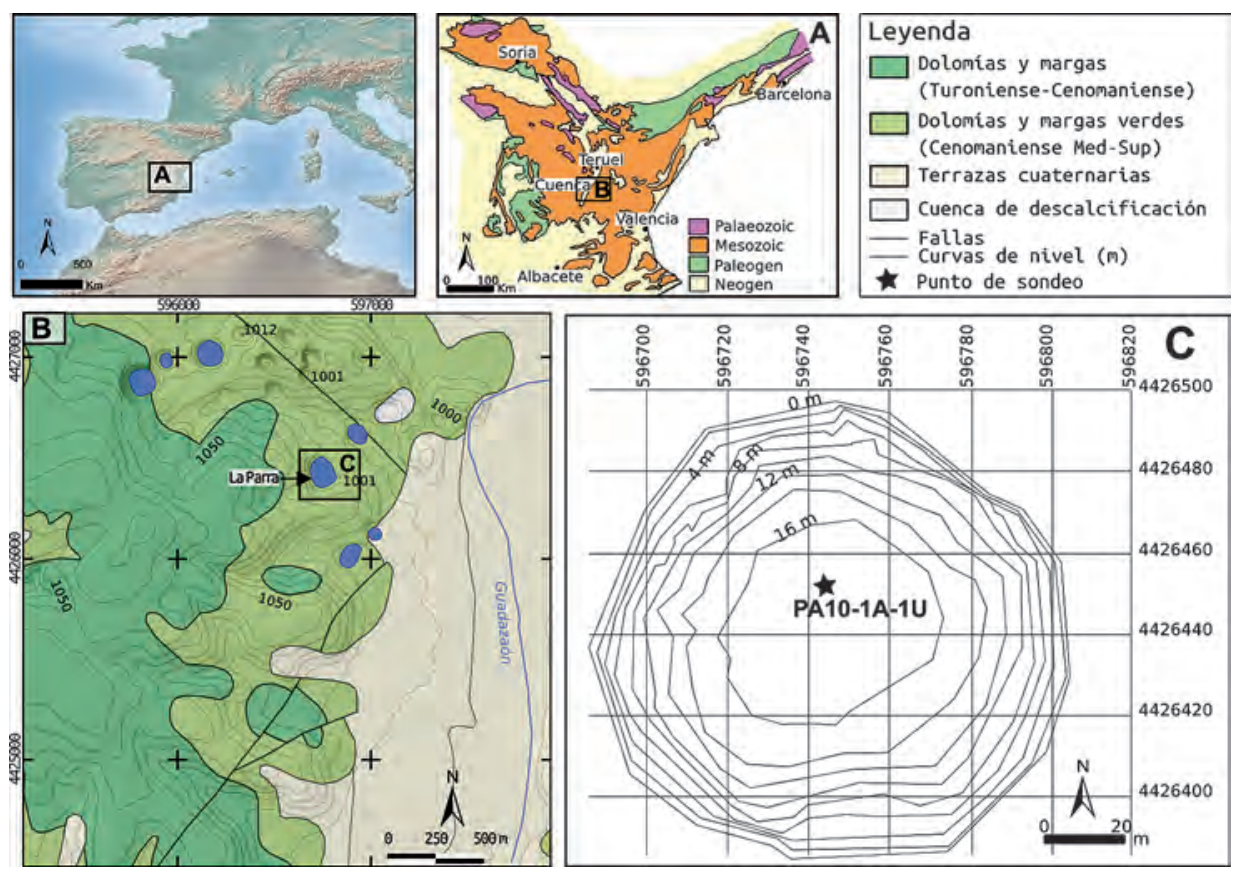

Figura 1. A) Localización de la zona de estudio y esquema geológico de la Cordillera Ibérica. B) Mapa geológico detallado del entorno de la laguna de La Parra (ver leyenda en la esquina superior derecha, basado en Ramírez del Pozo et al., 1973 y mapa topográfico nacional, IGN, 2002). C) Batimetría de la laguna y punto de toma del sondeo estudiado (estrella).

pH de 8. La composición isotópica de las aguas es similar en superficie y en el fondo por lo que corrobora la naturaleza holomíctica de la laguna. Por otra parte, los valores relativamente más positivos que los del acuífero y las aguas superficiales (Carmona y Bitzer, 2001) sugieren una evolución con respecto al acuífero por procesos de evaporación.

\section{Metodología}

En mayo de 2010 se recuperó un sondeo de 693 cm de longitud (PA10-1A-1U) en la parte más profunda de la laguna de La Parra $(17.5 \mathrm{~m})$ con la plataforma de sondeos flotante UWITEC ${ }^{\circledR}$ del IPE-CSIC. Posteriormente, en verano de 2011 se tomaron 21 sondeos cortos distribuidos en una malla de muestreo que cubre todo el lago.

Los sondeos se cortaron en dos mitades, se fotografiaron con la cámara CCD acoplada al escáner de fluorescencia de la Universidad de Barcelona y se definieron las facies sedimentarias en base a la inspección visual macroscópica, incluyendo color, tamaño de grano, estructuras sedimentarias, contenido fósil y también la observación de frotis de sedimento al microscopio óptico. Para el estudio microscópico de las facies sedimentarias se siguió la metodología descrita por Schnurrenberger et al. (2003) y se 
completó con microscopía electrónica de barrido (alto vacío) usando un JEOL JSM6400 de la Universidad de Zaragoza acoplado a un sensor EDAX INCA 300 X-Sight para la identificación elemental.

El sondeo largo (PA10-1A-1U) se muestreó cada $2 \mathrm{~cm}$ para analizar el carbono orgánico total (TOC) y el carbón inorgánico (TIC); cada $6 \mathrm{~cm}$ para nitrógeno total (TN); y cada $10 \mathrm{~cm}$ para análisis mineralógicos. El TOC y el TIC se midieron en el LECO SC144 DR del Instituto Pirenaico de Ecología (CSIC), mientras que el TN se midió en el LECO TRUSPEC CN del Centro de Edafología y Biología Aplicada del Segura (CSIC).

La mineralogía se analizó con un difractómetro de rayos X Siemens D-500 (Cu ka, $40 \mathrm{kV}, 30 \mathrm{~mA}$ con monocromador de grafito) y la identificación mineral y abundancia relativa se determinó a partir de los picos de intensidad siguiendo el procedimiento descrito por Chung (1974a, b).

La cronología para la secuencia lacustre se basa en la interpolación lineal de siete dataciones de macrorrestos vegetales terrestres por ${ }^{14} \mathrm{C}$ AMS del sondeo PA10-1A-1U, analizadas en el Poznan Radiocarbon Laboratory (Polonia) y calibradas usando el software CALIB REV 6.0.0 (Stuiver y Reimer, 1993) y la curva de calibración INTCAL09

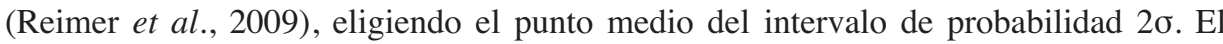
registro sedimentario abarca desde los $1639 \pm 77$ cal. yr. BP (coincidiendo con la etapa final del Periodo Húmedo Ibero-Romano) hasta el presente (Fig. 2).

\section{Resultados}

\subsection{El medio sedimentario reciente}

El estudio de las facies sedimentarias de los primeros centímetros de los 21 sondeos cortos ha permitido diferenciar cuatro ambientes de depósito modernos en la cuenca de la laguna: (i) una plataforma litoral con vegetación que estabiliza el sustrato, afectada por procesos de caída de rocas del escarpe carbonatado; (ii) una zona de talud escarpado (2-10 m de profundidad), que constituye un límite biológico a la vegetación por falta de luz y donde se pueden generar pequeños movimientos de masa por desestabilización; los sedimentos en la zona de talud están formados por limos y arenas con abundantes clastos carbonatados y restos vegetales; (iii) una zona transicional distal (10-16 m de profundidad) amplia (> $80 \mathrm{~m}$ anchura) caracterizada por una morfología plana y la presencia de limos masivos; y (iv) una pequeña zona distal alargada (16-17 $\mathrm{m}$ de profundidad) compuesta por limos finos masivos oscuros con abundante materia orgánica que comprende la parte central más profunda del lago.

\subsection{La secuencia sedimentaria}

La identificación e interpretación de las diferentes facies del registro sedimentario combina las observaciones sedimentológicas y microscópicas con los datos geoquímicos y mineralógicos. El sondeo largo PA10-1A-U está compuesto principalmente por 
limos detríticos carbonatados masivos y bandeados con diferentes proporciones de materia orgánica y de restos orgánicos y con intercalaciones de arenas y gravas. Se han diferenciado 11 facies (Barreiro-Lostres, 2012). La mayoría de las facies son detríticas y proceden de la erosión de materiales carbonatados de la cuenca de recepción, arrastrados por corrientes efímeras y escorrentía superficial, retrabajados por procesos litorales y depositados en la zona central del lago.

Las facies más abundantes son limos finos grises depositados por procesos de transporte de baja energía en la parte central de la laguna, lejos de la influencia de la escorrentía superficial y de los procesos litorales de retrabajamiento (oleaje, etc). La alternancia de capas claras y oscuras de estos limos implicaría cambios en las condiciones redox, más reductoras durante la sedimentación de los limos oscuros. Los niveles de limos gruesos presentan texturas granodecrecientes con una base irregular erosiva que indican procesos "turbidíticos" capaces de transportar los sedimentos desde la zona litoral hacia el centro del lago.

Las facies de arenas gruesas son típicas de la zona transicional y litoral, depositadas por procesos de escorrentía superficial y retrabajamiento litoral de menor (arenas finas) a mayor (arenas gruesas) energía. La presencia puntual de facies de gravas en la secuencia se interpreta como resultado de procesos de transporte tractivos de alta energía iniciados en la cuenca de recepción de la laguna y canalizados por un pequeño barranco situado al SO, con posible retrabajamiento del sedimento de las zonas más someras del lago. El depósito de facies gruesas (gravas y arenas) en las zonas más centrales del lago sugiere una intensificación de la escorrentía en la cuenca y, complementariamente, niveles del lago más bajos y predominio de condiciones litorales en toda la laguna.

Las facies de limos finos laminados contienen láminas blanquecinas de calcita autigénica compuestas por cristales calcíticos de 3-5 $\mu \mathrm{m}$ formados en el epilimnion durante el clímax de desarrollo algal en verano y láminas grises oscuras, compuestas por materia orgánica y silicatos depositados en otoño e invierno; ambas láminas tienen aprox. $1 \mathrm{~mm}$ de espesor. Estas facies son similares a las laminitas carbonatas encontradas en otros lagos kársticos de la Península Ibérica (Zoñar, Martín-Puertas et al., 2008; Arreo, Corella et al., 2010a; Montcortès, Corella et al., 2010b; La Cruz, Romero-Viana et al., 2008). Este tipo de laminación está asociada a ambientes de depósito de muy baja energía, escasa influencia aluvial y se ve favorecida por procesos de estratificación de las aguas, generalmente en etapas con nivel alto del lago (Brauer et al., 2004). La formación de estos cristales de calcita se considera bioinducida al coincidir con una mayor actividad biológica y condiciones limnológicas adecuadas para la preservación de carbonatos endógenos.

Basándonos en las facies sedimentarias, la secuencia de La Parra se ha dividido en cinco unidades sedimentológicas (Unidades I a V, Fig. 2).

La base de la secuencia está formada por margas verdes cenomanienses con evidencias de alteración kárstica (texturas de disolución y brechificación). La matriz está formada por clastos carbonatados angulares homométricos de 50-70 $\mu \mathrm{m}$ y granos de cuarzo de 80-100 $\mu \mathrm{m}$. 

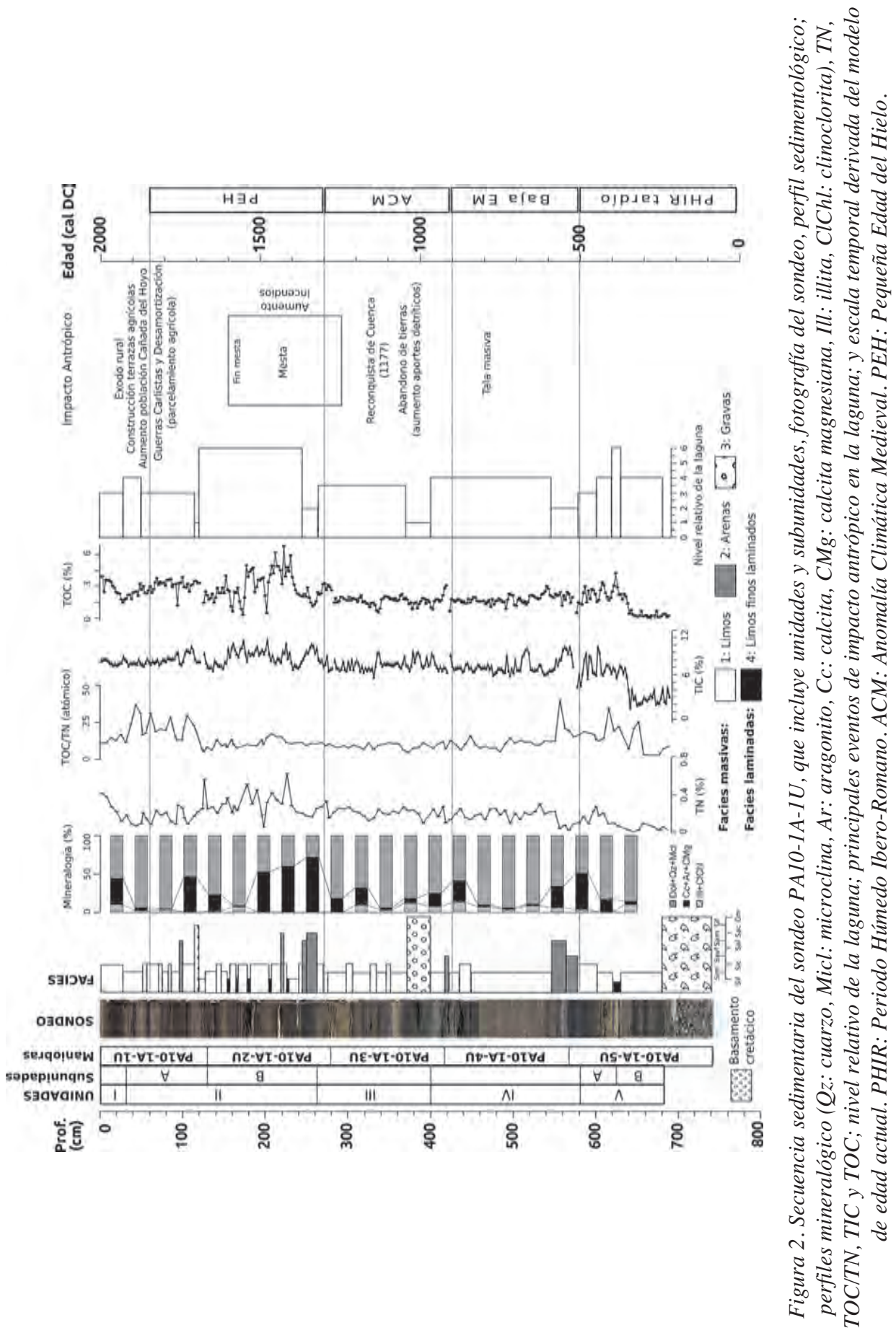
- La Unidad V (680-580 cm) marca el inicio de la sedimentación lacustre y está constituida por una secuencia granocreciente de limos marrones finos en la base (subunidad V B) y limos oscuros gruesos a techo (subunidad V A). A mitad del tramo una intercalación de limos finamente laminados marca la separación entre dos subunidades. La subunidad inferior V B $(680-625 \mathrm{~cm})$ está dominada por minerales detríticos (dolomita, silicatos) y presenta los valores más bajos de TIC y TOC de toda la secuencia. Los valores de TOC/TN muestran los valores más bajos en la base de esta sub-unidad y aumentan rápidamente hacia techo; la subunidad superior V A $(625-580 \mathrm{~cm})$, está caracterizada por mayor presencia de carbonatos endógenos (calcita y, secundariamente aragonito y calcita magnesiana) y valores relativamente altos de TIC, TOC y TN.

- La Unidad IV (580-400 cm) está formada por arenas medias y gruesas en la base, seguidas de limos medios. El contenido mineral está dominado por minerales de origen detrítico. Los valores de TN, TOC, TIC y TOC/TN son bajos.

- La Unidad III (400-260 cm) consiste en una alternancia de limos medios y gruesos con gravas en la base. Los minerales de origen detrítico siguen siendo dominantes.

- La Unidad II (260-30 cm) es la que presenta una mayor variedad de facies, desde arenas gruesas hasta limos, siendo característica la intercalación de limos finos laminados en capas de $5 \mathrm{~cm}$ de potencia. Presenta el contenido más alto de minerales carbonatados endógenos (calcita + aragonito + calcita magnesiana) de la serie. Se puede dividir en dos subunidades, la inferior II B (260-125 cm) está constituida por la alternancia de limos gruesos y facies finamente laminadas con los valores más altos de TN $(\sim 0.6 \%)$ y de TOC $(\sim 6 \%)$ y los valores más bajos de TOC/TN ( 9) de toda la serie. La subunidad II A $(125-30 \mathrm{~cm})$ está formada por una alternancia de limos grises claros de tamaño de grano medio, y oscuros más gruesos con alguna intercalación de arenas gruesas y gravas. Presenta valores de TN relativamente bajos $(\sim 0.1 \%)$, un descenso del TOC y los mayores valores de TOC/TN de toda la secuencia.

- La Unidad I (30-0 cm) está constituida por limos oscuros gruesos, mineralógicamente caracterizada por un contenido menor en minerales detríticos (Dolomita + Cuarzo + Feldespatos) que la subunidad II A y geoquímicamente por una tendencia ascendente del TOC y descendente de TOC/TN.

A lo largo de toda la secuencia, los valores de TIC muestran escasa variabilidad (en torno al 6\%), con los valores más bajos en la subunidad V B, y los valores relativamente más elevados en la parte basal de la unidad IV y la subunidad II B. En cuanto a la mineralogía, la fracción carbonatada es el componente principal en los sedimentos. La mayoría de estos carbonatos (dolomita, calcita) son de origen detrítico y proceden de formaciones carbonatadas del entorno de la laguna de La Parra. Los minerales exógenos (principalmente dolomita, cuarzo, minerales de la arcilla y feldespatos) son relativamente constantes en la secuencia y alcanzan los mayores valores en las facies limosas. 
Los minerales autigénicos (mayoritariamente calcita, secundariamente aragonito y calcita magnesiana) aparecen en cinco intervalos de facies de limos y arenas (base y techo Unidad IV, base y techo Subunidad II B, y Unidad I).

El contenido en materia orgánica (\% TOC) y sus características (TOC/TN) muestran cambios significativos a lo largo de la secuencia. El perfil de TOC sigue una tendencia similar al de TIC, con una media en torno al 3\%, pero alcanza los valores más altos (6$7 \%$ ) coincidiendo con la sedimentación de láminas finas de calcita endógena durante la subunidad II B y en épocas recientes (4.5\%, Unidad I). El TN sigue una tendencia diferenciada a la del TOC, oscilando entre el 0.1 y el 0.7 , lo que sugiere distintas fuentes de materia orgánica. Los valores más elevados (mayor proporción de materia orgánica de origen lacustre) coinciden con la sedimentación de facies finas laminadas en la subunidad II B, cuando las condiciones para la conservación de la materia orgánica eran óptimas debido al dominio de condiciones anóxicas en el fondo del lago, coincidiendo con menor entrada de material exógeno al lago (menor dilución sedimentaria de la MO).

La relación TOC/TN es variable ( 3 a 40), aunque relativamente baja a lo largo de toda la secuencia, lo que sugiere un predominio del origen lacustre de la materia orgánica (Meyers y Lallier-Vergès, 1999). Solo en dos intervalos - de 650 a 550 cm (380630 DC) y de 120 a $30 \mathrm{~cm}$ (1700-1920 DC) - los valores son > 15 sugiriendo una mayor influencia de materia orgánica vegetal terrestre del entorno de la laguna. En épocas recientes el TOC/TN vuelve a presentar valores relativamente bajos.

\section{Discusión}

A partir de las facies y de los análisis geoquímicos y mineralógicos (Fig. 2) se pueden establecer cinco etapas principales en la historia sedimentaria de la laguna de La Parra, durante los últimos 1600 años. Cada etapa, excepto la más antigua y la más reciente, se inicia con el depósito de facies gruesas y continúa con facies de limos (Fig. 2).

i. El primer episodio (300-500 DC) comienza con la inundación de la cuenca de La Parra, tras un aumento rápido del nivel piezométrico de la zona y la sedimentación de material detrítico fino, poco carbonatado y prácticamente sin materia orgánica. Las características peculiares de estos sedimentos (bajo TIC y TOC) sugieren que el material originario podría ser producto de los procesos kársticos de descalcificación que afectaron a la dolina seca de La Parra durante el periodo árido anterior. El aumento del TIC y el TOC en los sedimentos (350 DC) marca el desarrollo de condiciones propiamente lacustres en la dolina, con el depósito de limos grises oscuros y laminados que indican el rápido desarrollo de ambientes de sedimentación relativamente profundos durante la etapa final (380-420 DC). Esta etapa final sugiere niveles del lago relativamente elevados que corresponderían con el final del Periodo Húmedo Ibero-Romano. Los valores relativamente altos de TOC/TN indican aportes significativos de materia orgánica terrestre y sugieren una fase de erosión de suelos de la cuenca de recepción que pudiera estar relacionada con un mayor impacto antrópico durante el final de la época romana y el 
comienzo de la dominación visigoda, como talas masivas identificadas en el cercano registro de la laguna de La Cruz (Julià et al., 1998).

ii. La segunda etapa (500-980 DC), correspondiente a la Baja Edad Media, se inicia con un drástico cambio limnológico con la sedimentación de arenas gruesas en el centro del lago, que sugieren un aumento del transporte de sedimentos al lago desde la cuenca de drenaje así como, probablemente, un descenso en el nivel del lago. Tras esta fase relativamente corta, vuelve a dominar la sedimentación de limos en un ambiente relativamente más profundo, aunque los aportes terrígenos exógenos seguirían siendo elevados (altos \% de dolomita y silicatos) pero con menores aportes de materia orgánica exógena $(\mathrm{TOC} / \mathrm{TN}<12) \mathrm{y}$, por consiguiente una reducción en la erosión de los suelos en la cuenca.

iii. La tercera etapa (980-1350 DC) comienza con el depósito del mayor paquete de gravas de toda la secuencia indicando ambientes someros incluso en el centro del lago. Durante todo este periodo aumenta la presencia de facies de limos gruesos respecto a la etapa anterior, indicando un ambiente ligeramente menos profundo. El aumento de minerales carbonatados endógenos hacia el techo y el ligero aumento del TOC podrían indicar condiciones más adecuadas para la precipitación de calcita en el epilimnion, pero sin desarrollo de facies laminadas. Esta etapa engloba la Anomalía Climática Medieval (ACM).

iv. El cuarto estadio (1350-1950 DC) comienza con el depósito de facies de arenas y está caracterizado por el depósito de limos finamente laminados durante toda la secuencia, intercalados entre limos gruesos (subunidad II B, 1400-1700 DC), un aumento de TOC y TN y la presencia de minerales endógenos. En esta etapa, el ambiente de depósito correspondería a un lago profundo con frecuentes condiciones anóxicas en el fondo y una mayor actividad biológica. Este estadio corresponde a la Pequeña Edad del Hielo (PEH). De un modo similar, tanto en la cercana laguna de La Cruz como en el Lagunillo del Tejo se reconstruye un clima más húmedo para la PEH. Así, en La Cruz (Julià et al., 1998; RomeroViana, 2010) también se ha descrito la sedimentación de facies laminadas, procesos meromícticos y alto nivel de agua, mientras en el Lagunillo del Tejo (López-Blanco et al., 2011) se reconstruyen niveles del lago más altos durante esta etapa. El desarrollo de las condiciones meromícticas en estos lagos podría estar relacionado con factores climáticos (mayor precipitación, temperaturas más frías y niveles más altos de los lagos), antrópicos, como la expansión de la mesta (trashumancia) en la zona, facilitando los aportes detríticos de finos por roturación y quema de tierras para pastos, y el aumento de la bioproductividad en los lagos (Julià et al., 1998).

Hacia el final de este periodo (unidad II A, entre 1700-1850 DC) domina la sedimentación de gravas, arenas y limos gruesos y se registran los valores más elevados de TOC/TN, indicativos de intensa erosión de los suelos en la cuenca. Esta fase de mayor erosión correspondería con la de mayor impacto humano en la cuenca e incluiría incendios durante las Guerras Carlistas y cambios de usos 
del suelo tras la Desamortización al propiciar el parcelamiento agrícola, el aumento en la población de Cañada del Hoyo y la construcción de terrazas agrícolas en la zona de lagos (López-Blanco et al., 2011). Aunque la sedimentación de limos más finos sugiere una recuperación de los niveles hidrológicos durante el siglo XIX, los elevados valores de la relación TOC/TN testifican el elevado impacto humano en la cuenca hasta mediados del siglo XX. En el Lagunillo del Tejo se observa también un aumento en la tasa de sedimentación durante el siglo XIX y comienzo del XX, coincidiendo en este caso con niveles del lago más bajos (Romero-Viana et al., 2009; López-Blanco et al., 2011).

v. Durante la quinta etapa (1950-2010 DC) se produce un descenso rápido en la entrada de material detrítico y orgánico acompañado de un aumento de la bioproductividad de la laguna (aumento de TOC y TN), posiblemente debido al éxodo rural y abandono de las áreas de cultivo. La ausencia de facies laminadas indica que durante este periodo han predominado condiciones óxicas en el fondo de la laguna.

Los principales estadios hidrológicos y climáticos identificados en La Parra son coherentes con las reconstrucciones disponibles en la Península Ibérica, tanto locales como regionales. En general, los últimos milenios están caracterizados por: i) un periodo húmedo contemporáneo a la época romana de importancia regional, aunque con una gran variabilidad, descrito desde el sur (Martín-Puertas et al., 2008) hasta el NE de España (lago de Estanya, Morellón et al., 2008; lago de Moncortés, Corella et al., 2010) y también en la zona central (Somolinos, Currás et al., 2012; Laguna de la Cruz, Julià et al., 1998). En La Parra, este aumento de humedad ocurrió al final de dicho periodo (300-500 DC), contrariamente al sur de la Península donde este periodo húmedo fue más prolongado (500 AC-500 DC, Martín-Puertas et al., 2008) ii) un periodo más árido correspondiente a la ACM (500-1400 DC), coincidente con niveles del lago más bajos y un aumento de aportes detríticos. Similares características se observan en Moncortés (Corella et al., 2010) y Estanya (Morellón et al., 2008) y en numerosos registros continentales y marinos de toda la Península (Moreno et al., 2012) y iii) fases húmedas durante la PEH (1450 - 1650 DC) caracterizadas por niveles hidrológicos altos y el desarrollo de facies laminadas con carbonatos endógenos. Estas fases húmedas dentro de la PEH han sido descritas en otros registros continentales ibéricos, aunque dentro de una amplia variabilidad hidrológica y climática (Morellón et al., 2008, 2012).

\section{Conclusiones}

La secuencia sedimentaria de la laguna de La Parra representa el registro más largo (6.93 m, últimos 1600 años) y mejor datado (nueve muestras de ${ }^{14} \mathrm{C}$ ) obtenido en el complejo lacustre de las Torcas de Cañada del Hoyo.

La secuencia sedimentaria de La Parra es característica por su alta variabilidad de facies (11 en total), constituida principalmente por sedimentos carbonatados con diferente contenido en materia orgánica, y está controlada por fluctuaciones en los aportes 
detríticos y el balance hidrológico durante los últimos 1600 años. La reconstrucción sedimentaria e hidrológica basada en análisis sedimentológicos, geoquímicos y mineralógicos ha permitido la identificación de cinco unidades sedimentarias y cinco episodios paleohidrológicos coherentes con otros registros paleoclimáticos de la Península Ibérica, mostrando fases áridas (anterior al periodo romano y durante la $\mathrm{ACM}$ ) y más húmedas (final del periodo romano y la Pequeña Edad del Hielo). La historia hidrológica de la laguna empieza con (i) un aumento del nivel piezométrico y el inicio de la sedimentación lacustre hacia el año 300 DC; posteriormente (ii) tras una breve etapa somera, aumentan progresivamente los niveles durante la Baja Edad Media (500-900 DC); seguido (iii) por niveles del lago relativamente bajos durante la ACM (1000-1300) con un elevado aporte de material detrítico y erosión de suelos; después (iv) prosigue con un periodo de niveles lacustres altos y desarrollo de condiciones anóxicas frecuentes coincidiendo con la PEH (1300-1850 DC); y por último (v) fluctuaciones hidrológicas durante el siglo XX, comenzando con un ligero aumento de los niveles a principios de siglo y tendencia hacia un descenso relativo en tiempos recientes (1950-actualidad). Aunque el complejo de lagunas de Cañada del Hoyo está actualmente protegido, el acuífero kárstico regional está siendo explotado para usos agrícolas.

Los mayores aportes detríticos en la laguna de La Parra durante la Edad Media y la Edad Contemporánea producidos por los cambios socioeconómicos (principalmente la trashumancia y roturación de bosques) y climáticos son coherentes con las reconstrucciones realizadas en otros lagos del complejo de las Torcas.

\section{Agradecimientos}

Esta investigación se ha financiado con el proyecto GLOBALKARST (CGL200908415), concedido por la Comisión Interministerial de Ciencia y Tecnología (CICYT). Fernando Barreiro tiene una beca predoctoral concedida por el programa JAE del CSIC. Un especial agradecimiento a las personas que intervinieron en las campañas de campo y a los servicios de laboratorio del IPE-CSIC.

\section{Referencias bibliográficas}

Alonso, F. 1986. Karst Externo. Las torcas de Cuenca. En Atlas De Geomorfología, E. Martínez de Pisón, B. Tello (eds.), Alianza, Madrid pp. 273-284.

Barreiro-Lostres, F. 2012. Depositional evolution of La Parra karstic lake (Iberian Chain, Spain) during the last 1,600 years: Climate and human impact implications. Trabajo fin de Máster. Universidad de Zaragoza, Zaragoza, $39 \mathrm{pp}$.

Brauer, A. 2004. Annually laminated lake sediments and their palaeoclimatic relevance. En The Climate in Historical Times. Towards a Synthesis of Holocene Proxy Data and Climate Models, H. Fischer, T. Kumke, G. Lohmann, G. Floser, H. Miller, V. Von Storch, J.F.W. Negendank (eds.), Springer, Berlin, pp. 109-129.

Carmona, J.M., Bitzer, K. 2001. Los sistemas cársticos de Lagunas de Cañada del Hoyo y Torcas de los Palancares (Serranía de Cuenca. España). En Las Caras Del Agua Subterránea, Instituto Geológico y Minero de España, Madrid, pp. 451-460. 
Chung, F.H. 1974a. Quantitative interpretation of X-ray diffraction patterns of mixtures. II. Adiabatic principle of X-ray diffraction analysis of mixtures. Journal of Applied Crystallography 7, 526-531.

Chung, F.H. 1974b. Quantitative interpretation of X-ray diffraction patterns of mixtures. I. Matrixflushing method for quantitative multicomponent analysis. Journal of Applied Crystallography 7, 519-525.

Corella, J.P., Amrani, A.E., Sigró, J., Morellón, M., Rico, E., Valero-Garcés, B.L. 2010a. Recent evolution of Lake Arreo, northern Spain: influences of land use change and climate. Journal of Paleolimnology 46, 469-485.

Corella, J.P., Moreno, A., Morellón, M., Rull, V., Giralt, S., Rico, M.T., Pérez-Sanz, A., ValeroGarcés, B.L. 2010b. Climate and human impact on a meromictic lake during the last 6,000 years (Montcortès Lake, Central Pyrenees, Spain). Journal of Paleolimnology 46, 351-367.

Corella, J.P., Brauer, A., Mangili, C., Rull, V., Vegas-Villarrubia, T., Morellón, M., Valero-Garcés, B.L. 2012. The 1.5-ka varved record of Lake Montcortès (southern Pyrenees, NE Spain) Quaternary Research 78, 323-332.

Currás, A., Zamora, L., Reed, J.M., García-Soto, E., Ferrero, S., Armengol, X., Mezquita-Joanes, F., Marqués, M.A., Riera, S., Julià, R. 2012. Climate change and human impact in central Spain during Roman times: High-resolution multi-proxy analysis of a tufa lake record (Somolinos, $1280 \mathrm{~m}$ asl). Catena 89, 31-53.

Eraso, A.E., López-Azevedo, V., López, M., Navarro, V., Suso, J., Santos, V. 1979. Estudio de Las Torcas de Palancares y Cañada del Hoyo en el karst de la Serranía de Cuenca. Kobie 9, 7-79.

Gil, J., Carenas, B., Segura, M., García-Hidalgo, J.F., García, A. 2004. Revisión y correlación de las unidades litoestratigráficas del Cretácico Superior en la región central y oriental de España. Revista de la Sociedad Geológica de España 17, 249-266.

González-Sampériz, P., Valero-Garcés, B.L., Moreno, A., Morellón, M., Navas, A., Machín, J., Delgado-Huertas, A. (2008). Vegetation changes and hydrological fluctuations in the Central Ebro Basin (NE Spain) since the Late Glacial period: Saline lake records. Palaeogeography, Palaeoclimatology, Palaeoecology 259, 157-181.

Höbig, N., Weber, M.E., Kehl, M., Weniger, G.C., Julià, R., Melles, M., Fülöp, R.H., Vogel, H., Reicherter, K. 2012. Lake Banyoles (northeastern Spain): A Last Glacial to Holocene multiproxy study with regard to environmental variability and human occupation. Quaternary International 274, 205-218.

Julià, R., Burjachs, F., Dasi, M., Mezquita, F., Miracle, M., Roca, J., Seret, G., Vicente, E. 1998. Meromixis origin and recent trophic evolution in the Spanish mountain lake La Cruz. Aquatic Sciences 60, 279-299.

López-Blanco, C., Gaillard, M.J., Miracle, M.R., Vicente, E. 2011. Lake-level changes and fire history at Lagunillo del Tejo (Spain) during the last millennium: Climate or humans? The Holocene 22, 551-560.

López-Merino, L., Moreno, A., Leira, M., Sigró, J., González-Sampériz, P., Valero-Garcés, B.L., López-Sáez, J.A., Brunet, M., Aguilar, E. 2011. Two hundred years of environmental change in Picos de Europa National Park inferred from sediments of Lago Enol, northern Iberia. Journal of Paleolimnology 46, 453-467.

Martín-Puertas, C., Valero-Garcés, B.L., Mata, M.P., González-Sampériz, P., Bao, R., Moreno, A., Stefanova, V. 2008. Arid and humid phases in southern Spain during the last 4000 years: the Zonar Lake record, Cordoba. The Holocene 18, 907-921.

Martín-Puertas, C., Valero-Garcés, B.L., Mata, M.P., Moreno, A., Giralt, S., Martínez-Ruiz, F., Jiménez-Espejo, F. 2009. Geochemical processes in a Mediterranean Lake: a high-resolution study of the last 4,000 years in Zoñar Lake, southern Spain. Journal of Paleolimnology 46, 405-421. 
Meyers, P., Lallier-Vergés, E. 1999. Lacustrine Sedimentary Organic Matter Records of Late Quaternary Paleoclimates. Journal of Paleolimnology 21, 345-372.

Morellón, M., Valero-Garcés, B., Moreno, A., González-Sampériz, P., Mata, P., Romero, O., Maestro, M., Navas, A. 2008. Holocene palaeohydrology and climate variability in northeastern Spain: The sedimentary record of Lake Estanya (Pre-Pyrenean range). Quaternary International 181, 15-31.

Morellón, M., Valero-Garcés, B., González-Sampériz, P., Vegas-Villarrúbia, T., Rubio, E., Rieradevall, M., Delgado-Huertas, A., Mata, P., Romero, Ó., Engstrom, D.R., López-Vicente, M., Navas, A., Soto, J. 2011. Climate changes and human activities recorded in the sediments of Lake Estanya (NE Spain) during the Medieval Warm Period and Little Ice Age. Journal of Paleolimnology 46, 423-452.

Morellón, M., Pérez-Sanz, A., Corella, J.P., Büntgen, U., Catalán, J., González-Sampériz, P., González-Trueba, J.J., López-Sáez, J.A., Moreno, A., Pla-Rabes, S., Saz-Sánchez. M.A., Scussolini, P., Serrano, E., Steinhilber, F., Stefanova, V., Vegas-Villarrúbia, T., Valero-Garcés, B. 2012. A multi-proxy perspective on millennium-long climate variability in the Southern Pyrenees. Climate of the Past 8, 683-700.

Moreno, A., Valero-Garcés, B.L., González-Sampériz, P., Rico, M. 2008. Flood response to rainfall variability during the last 2000 years inferred from the Taravilla Lake record (Central Iberian Range, Spain). Journal of Paleolimnology 40, 943-961.

Moreno, A., López-Merino, L., Leira, M., Marco-Barba, J., González-Sampériz, P., Valero-Garcés, B.L., López-Sáez, J.A., Santos, L., Mata, P., Ito, E. 2009. Revealing the last 13500 years of environmental history from the multiproxy record of a mountain lake (Lago Enol, northern Iberian Peninsula). Journal of Paleo-limnology 46, 327-349.

Moreno, A., Pérez, A., Frigola, J., Nieto-Moreno, V., Rodrigo-Gámiz, M., Martrat, B., GonzálezSampériz, P., Morellón, M., Martín-Puertas, C., Corella, J.P. 2012. The Medieval Climate Anomaly in the Iberian Peninsula reconstructed from marine and lake records. Quaternary Science Reviews 43, 16-32.

Ramírez del Pozo, J., Portero, J.M., Olivé, A. 1973. Mapa Geológico de España y Memoria, Hoja $n^{\circ} 635$ (Fuentes). IGME, Madrid.

Reimer, P.J., Baillie, M.G.L., Bard, E., Bayliss, A., Beck, J.W., Blackwell, P.G., Bronk-Ramsey, C. 2009. INTCAL 09 and MARINE09 radiocarbon age calibration curves, 0-50,000 years Cal BP. Radiocarbon 51, 1111-1150.

Roberts, N., Moreno, A., Valero-Garcés, B.L., Corella, J.P., Jones, M., Allcock, S., Woodbridge, J., Morellón, M., Luterbacher, J., Xoplaki, E., Türkes, M. 2012. Palaeolimnological evidence for an east-west climate see-saw in the Mediterranean since AD 900. Global and Planetary Change 84-85, 23-34.

Rodrigo, F.S., Barriendos, M. 2008. Reconstruction of seasonal and annual rainfall variability in the Iberian Peninsula (16th-20th centuries) from documentary data. Global and Planetary Change 63, 243-257.

Romero-Viana, L., Julià, R., Camacho, A., Vicente, E., Miracle, M.R. 2008. Climate Signal in Varve Thickness: Lake La Cruz (Spain), a Case Study. Journal of Paleolimnology 40, 703-714.

Romero-Viana, L., Miracle, M.R., López-Blanco, C., Cuna, E., Vilaclara, G., García-Orellana, J., Keely, B.J., Camacho, A., Vicente, E. 2009. Sedimentary multiproxy response to hydroclimatic variability in Lagunillo del Tejo (Spain). Hydrobiologia 631, 231-245.

Romero-Viana, L., Julià, R., Schimmel, M., Camacho, A., Vicente, E., Miracle, M.R. 2011. Reconstruction of annual winter rainfall since A.D. 1579 in central-eastern Spain based on calcite laminated sediment from Lake La Cruz. Climatic Change 107, 343-361.

Schnurrenberger, D., Russell, J., Kelts, K. 2003. Classification of lacustrine sediments based on sedimentary components. Journal of Paleolimnology 29, 141-154. 
Stuiver, M., Reimer, P.J. 1993. Extended ${ }^{14} \mathrm{C}$ database and revised CALIB radiocarbon calibration program. Radiocarbon 35, 215-230.

Valero-Garcés, B.L., Moreno, A. 2011. Iberian lacustrine sediment records: responses to past and recent global changes in the Mediterranean region. Journal of Paleolimnology 46, 319-325. 